Francesca Da Lio · Boyan Sirakov

\title{
Symmetry results for viscosity solutions of fully nonlinear uniformly elliptic equations
}

Received November 25, 2004

Abstract. We study uniformly elliptic fully nonlinear equations

$$
F\left(D^{2} u, D u, u, x\right)=0,
$$

and prove results of Gidas-Ni-Nirenberg type for positive viscosity solutions of such equations. We show that symmetries of the equation and the domain are reflected by the solution, both in bounded and unbounded domains.

Keywords. Symmetry, fully nonlinear equations, viscosity solutions

\section{Introduction}

In this note we establish symmetry results of Gidas-Ni-Nirenberg type for viscosity solutions of the equation

$$
\begin{cases}F\left(D^{2} u, D u, u, x\right)=0 & \text { in } \mathcal{O}, \\ u>0 & \text { in } \mathcal{O}, \\ u=0 & \text { on } \partial \mathcal{O}\end{cases}
$$

where $\mathcal{O}$ is a domain in $\mathbb{R}^{n}, F$ is a continuous function defined on $\mathcal{S}^{n}(\mathbb{R}) \times \mathbb{R}^{n} \times \mathbb{R} \times \overline{\mathcal{O}}$ with values in $\mathbb{R}$ and $\mathcal{S}^{n}(\mathbb{R})$ denotes the space of real, $n \times n$, symmetric matrices. More precise assumptions on $F$ are given later on. The solution $u$ of this nonlinear problem is scalar and $D u, D^{2} u$ denote respectively the gradient and the Hessian matrix of $u$.

A model problem for 1.1 will be the equations

$$
\mathcal{M}_{\lambda, \Lambda}^{+}\left(D^{2} u\right)+f(u)=0, \quad \mathcal{M}_{\lambda, \Lambda}^{-}\left(D^{2} u\right)+f(u)=0,
$$

where $f$ is a locally Lipschitz continuous function and $\mathcal{M}_{\lambda, \Lambda}^{+}, \mathcal{M}_{\lambda, \Lambda}^{-}$are the extremal Pucci operators ([17], [10]), with parameters $0<\lambda \leq \Lambda$, defined by

$$
\mathcal{M}_{\lambda, \Lambda}^{+}(M)=\Lambda \sum_{e_{i}>0} e_{i}+\lambda \sum_{e_{i}<0} e_{i}, \quad \mathcal{M}_{\lambda, \Lambda}^{-}(M)=\lambda \sum_{e_{i}>0} e_{i}+\Lambda \sum_{e_{i}<0} e_{i}
$$

F. Da Lio: Dipartimento di Matematica, Università di Padova, Via Belzoni, 7, 35131 Padova, Italy; e-mail: dalio@math.unipd.it

B. Sirakov: MODALX, UFR SEGMI, Université de Paris 10, 92001 Nanterre Cedex, France, and CAMS, EHESS, 54 bd Raspail, 75006 Paris, France; e-mail: sirakov@ehess.fr

Mathematics Subject Classification (2000): 35B30, 35J60 
for any symmetric $n \times n$ matrix $M$. Here $e_{i}=e_{i}(M), i=1, \ldots, n$, denote the eigenvalues of $M$. Pucci's operators are extremal in the sense that $\mathcal{M}_{\lambda, \Lambda}^{+}(M)=\sup _{A \in \mathcal{A}_{\lambda, \Lambda}} \operatorname{tr}(A M)$, $\mathcal{M}_{\lambda, \Lambda}^{-}(M)=\inf _{A \in \mathcal{A}_{\lambda, \Lambda}} \operatorname{tr}(A M)$, where $\mathcal{A}_{\lambda, \Lambda}$ denotes the set of all symmetric matrices whose eigenvalues lie in the interval $[\lambda, \Lambda]$.

Let us recall the classical result of Gidas, Ni and Nirenberg [15], which states that positive $C^{2}$-solutions of the Dirichlet problem for the equation

$$
\Delta u+f(u)=0, \quad f \in C^{0,1}(\mathbb{R}),
$$

(this is equation (1.2) corresponding to $\lambda=\Lambda=1$ ) in a ball are necessarily radial, or more generally, if the domain is symmetric and convex with respect to a hyperplane then the solutions have the same symmetry. Related results for 1.3 in the whole space and exterior domains were obtained by C. Li [16], W. Reichel [22], and B. Sirakov [24], under the supplementary hypothesis that $f$ is nonincreasing in a right neighbourhood of zero.

Symmetry results in the spirit of Gidas, Ni and Nirenberg for classical solutions of fully nonlinear equations of type (1.1) were obtained by C. Li [16]. Extensions and simple proofs of these results are due to Berestycki and Nirenberg [7].

An essential hypothesis in [7] is that the operator $F$ is $C^{1}$ in the matrix of the second derivatives of $u \in C^{2}(\mathcal{O}) \cap C(\overline{\mathcal{O}})$. This prevents applying these results to important classes of equations, such as equations involving Pucci's operators, Bellman or Isaacs equations. On the other hand, the symmetry result was proved for viscosity solutions of [1.1, without differentiability assumption on $F$, by Badiale [2] (see also Badiale-Bardi [3] for results on general first order equations), under the hypothesis that the operator $F$ satisfies a comparison principle. This is a quite strong assumption, which essentially requires that the operator $F$ is nonincreasing with respect to the $u$ variable or at least convex in the $\left(D u, D^{2} u\right)$ variables (cf. Section 5 in [13]).

It is our purpose here to join together and extend the above quoted results. We are going to show that the moving planes method of Alexandrov [1] and Serrin [23], in its version developed in [7], can actually be adapted to work in the setting of viscosity solutions and general equations (1.1).

Before proceeding to the precise statements, let us recall that existence and uniqueness of viscosity solutions of boundary value problems of type (1.1) has been very extensively studied for proper operators, that is, when $F$ is nonincreasing in $u$ : see for example [13], [11], [12]. Quite recently existence of solutions of nonproper equations of type (1.2) was established by Felmer-Quaas [14] and Quaas-Sirakov [19]. In particular, in [14] it was shown that the Dirichlet problem for (1.2) in a ball has a positive radial solution when $f$ has some power growth at infinity. It follows from our result that actually any positive solution is radial. It is well known that proving symmetry for solutions is an important step towards proving uniqueness in the nonproper case.

Next we list our assumptions on the nonlinearity $F$.

(H1) (Regularity) For all $R>0$ there exists a constant $K_{R}>0$ and a function $\omega_{R}$ : $\mathbb{R}^{+} \rightarrow \mathbb{R}^{+}$, with $\omega_{R}\left(0^{+}\right)=0$ such that, for any $x, y \in \overline{\mathcal{O}}, p, q \in \mathbb{R}^{n}, M, N \in$ $\mathcal{S}^{n}(\mathbb{R}), u_{1}, u_{2} \in[-R, R]$, 


$$
\begin{aligned}
\left|F\left(M, p, u_{1}, x\right)-F\left(N, q, u_{2}, y\right)\right| \leq & K_{R}\{|p-q|+\|M-N\| \\
& \left.+\left|u_{1}-u_{2}\right|+|x-y|(\|M\|+\|N\|)\right\} \\
& +\omega_{R}(|x-y|(1+|p|+|q|)) .
\end{aligned}
$$

(H2) (Uniform ellipticity) There exists $\kappa>0$ such that, for any $x \in \overline{\mathcal{O}}, u \in \mathbb{R}, p \in \mathbb{R}^{n}$, $M, N \in \mathcal{S}^{n}(\mathbb{R})$ with $N \geq 0$,

$$
F(M+N, p, u, x)-F(M, p, u, x) \geq \kappa \operatorname{tr}(N) .
$$

It is standard to show that $(H 1)$ and $(H 2)$ imply the following assumption (actually, $(H 1)-(H 2)$ reduce to $(H 3)$ when $F$ is independent of $x)$ :

(H3) For any $R>0$ there exists a constant $K_{R}>0$ such that for each $M, N \in \mathcal{S}^{n}(\mathbb{R})$, $p, q \in \mathbb{R}^{n}, x \in \overline{\mathcal{O}}, u, v \in[-R, R]$, we have

$$
\begin{aligned}
& F(M, p, u, x)-F(N, q, v, x) \geq \mathcal{M}_{\lambda, \Lambda}^{-}(M-N)-K_{R}(|p-q|+|u-v|), \\
& F(M, p, u, x)-F(N, q, v, x) \leq \mathcal{M}_{\lambda, \Lambda}^{+}(M-N)+K_{R}(|p-q|+|u-v|),
\end{aligned}
$$

with $\lambda, \Lambda$ depending on $K_{R}$ and $\kappa$.

Another example we have in mind is the standard quasilinear equation

$$
\operatorname{tr}\left[B(x) D^{2} u\right]+H(x, u, D u)=0 \quad \text { in } \mathcal{O},
$$

where $B$ is an $n \times n$ real symmetric matrix and $H$ a continuous function. In this case $(H 2)$ is satisfied if $B(x) \geq \kappa$ Id for all $x \in \mathcal{O}$, and $(H 1)$ is satisfied if

(i) $B$ is a bounded and locally Lipschitz continuous function of $x$;

(ii) the function $H$ satisfies: for any $R>0$, there are a constant $K_{R}>0$ and a function $\omega_{R}: \mathbb{R}^{+} \rightarrow \mathbb{R}^{+}$with $\omega_{R}\left(0^{+}\right)=0$ such that, for any $x, y \in \overline{\mathcal{O}}, p, q \in \mathbb{R}^{n}, u, v \in$ $[-R, R]$,

$\left|H\left(x, u_{1}, p\right)-H\left(y, u_{2}, q\right)\right| \leq \omega_{R}(|x-y|(1+|p|+|q|))+K_{R}(|p-q|+|u-v|)$.

For any matrix $M=\left(m_{i j}\right) \in \mathcal{S}^{n}(\mathbb{R})$ we denote by $M^{(k)}$ the matrix obtained from $M$ by replacing $m_{i k}$ and $m_{k j}$ by $-m_{i k}$ and $-m_{k j}$ respectively, for any $i \neq k, j \neq k$. Note that $M$ and $M^{(k)}$ always have the same eigenvalues.

For any vector $p \in \mathbb{R}^{n}$ we write $p^{(k)}=\left(p_{1}, \ldots, p_{k-1},-p_{k}, p_{k+1}, \ldots, p_{N}\right)$. We consider the following hypothesis.

$\left(O_{k}\right) \mathcal{O}$ is convex in the direction $x_{k}$, symmetric with respect to the hyperplane $\left\{x_{k}=0\right\}$, and for all $M \in \mathcal{S}^{n}(\mathbb{R}), p \in \mathbb{R}^{n}, u \in(0, \infty), x \in \mathcal{O}$,

$$
F(M, p, u, x)=F\left(M^{(k)}, p^{(k)}, u, x^{(k)}\right),
$$

and $F$ is nonincreasing in $x_{k}$ for $x_{k}>0$.

Then we have the following theorem. 
Theorem 1.1. Suppose $\mathcal{O} \subset \mathbb{R}^{n}$ is bounded, and assume (H1), (H2), and $\left(O_{k}\right)$ for some $k \in\{1, \ldots, N\}$. Let $u \in C(\overline{\mathcal{O}})$ be a viscosity solution of $(1.1)$. Then $u$ is symmetric in $x_{k}$, that is, $u(x)=u\left(x^{(k)}\right)$ for all $x \in \mathcal{O}$. In addition, $u$ is strictly decreasing in $x_{k}>0$.

Corollary 1.1. Suppose $\mathcal{O}$ is a ball centred at the origin, $F$ is radial in $x$ and nonincreasing in $|x|$, and satisfies $(H 1),(H 2)$, and $\left(O_{k}\right)$ for all $k \in\{1, \ldots, N\}$. Then every viscosity solution of $(1.1)$ is radial and strictly decreasing in $|x|$.

Next, we turn to symmetry in unbounded domains for autonomous equations.

In [4] Badiale and Bardi showed that positive solutions of a large class of (not necessarily uniformly) elliptic equations in $\mathbb{R}^{n}$ or exterior domains are asymptotically radial, that is, level sets of the solutions approach spheres as $|x|$ goes to infinity. The following theorems can be seen as completion of these results for uniformly elliptic equations and symmetric domains, for which we can show that all level sets are spheres.

Theorem 1.2. Assume $\left(O_{k}\right)$ for all $k \in\{1, \ldots, N\}$ and suppose $F$ does not depend on $x$, satisfies (H1)-(H2) and is nonincreasing in $u \in[0, \delta)$ for some $\delta>0$. Let $u \in C\left(\mathbb{R}^{n}\right)$ be a viscosity solution of

$$
\begin{cases}F\left(D^{2} u, D u, u\right)=0 & \text { in } \mathbb{R}^{n}, \\ u>0 & \text { in } \mathbb{R}^{n}, \\ u \rightarrow 0 & \text { as }|x| \rightarrow \infty .\end{cases}
$$

Then $u$ is radial and strictly decreasing in $|x|$.

The proof of Theorem 1.2 is a particular case of the proof of the following more general result on symmetry in exterior domains.

Theorem 1.3. Suppose $\mathcal{O}=\mathbb{R}^{n} \backslash B$ for some ball $B, F$ does not depend on $x$, satisfies $(H 1)-(H 2)$ and $\left(O_{k}\right)$ for all $k \in\{1, \ldots, N\}$, and $F$ is nonincreasing in $u \in[0, \delta)$ for some $\delta>0$. Let $u \in C(\overline{\mathcal{O}})$ be a viscosity solution of

$$
\begin{cases}F\left(D^{2} u, D u, u\right)=0 & \text { in } \mathcal{O}, \\ u>0 & \text { in } \mathcal{O}, \\ u=a & \text { on } \partial B, \\ u \rightarrow 0 & \text { as }|x| \rightarrow \infty\end{cases}
$$

for some $a>0$. Suppose in addition that for all $x \in \partial B$ and for all directions $v \in \mathbb{R}^{n} \backslash\{0\}$ such that $v \cdot n(x)>0$, where $n(x)=x /|x|$, we have

$$
\limsup _{t \searrow 0} \frac{u(x+t v)-u(x)}{t} \leq 0 .
$$

Then $u$ is radial and strictly decreasing in $|x|$. 


\section{Proofs}

We first show that under $(H 1)-(H 2)$ the difference of a lower semicontinuous supersolution and an upper semicontinuous subsolution of (1.1) is a supersolution of an equation involving a positively homogeneous uniformly elliptic operator.

In the following we will denote by $B U S C(\overline{\mathcal{O}})$ and $B L S C(\overline{\mathcal{O}})$ respectively the set of bounded upper and lower semicontinuous functions in $\overline{\mathcal{O}}$.

Proposition 2.1. Assume that $F$ satisfies $(H 1)-(H 2)$. Let $u_{1} \in B U S C(\overline{\mathcal{O}})$ and $u_{2} \in$ $B L S C(\overline{\mathcal{O}})$ be respectively a viscosity subsolution and a viscosity supersolution of $F\left(D^{2} u, D u, u, x\right)=0$ in $\mathcal{O}$. Then there exist positive constants $\lambda, \Lambda, b$ (depending on the $L^{\infty}$-norms of $u_{1}$ and $\left.u_{2}\right)$ and a bounded function $c(x)$ (whose $L^{\infty}$-norm depends only on the $L^{\infty}$-norms of $u_{1}$ and $u_{2}$, and on the local Lipschitz norm of $F$ with respect to $u$ on the ranges of $\left.u_{1}, u_{2}\right)$ such that the function $w=u_{2}-u_{1}$ is a viscosity supersolution of

$$
\mathcal{M}_{\lambda, \Lambda}^{-}\left(D^{2} w\right)-b|D w|+c(x) w=0 \quad \text { in } \mathcal{O} .
$$

If in addition $F$ is nonincreasing with respect to $u$ then $c \leq 0$ in $\mathcal{O}$.

We first make some comments. The strategy of the proof of Proposition 2.1 is similar to the one of the comparison principle for fully nonlinear operators (see [13]). A difficulty comes from $(H 1)-(H 2)$ and can be seen on a term like $\operatorname{tr}\left(B(x) D^{2} u\right)$ (in the case of quasilinear equations): in general, one assumes that $B$ has the form $B=\sigma \sigma^{T}$ for some Lipschitz continuous matrix $\sigma$ and the uniqueness proof uses $\sigma$ in an essential way, both in the degenerate and nondegenerate case. Here we want just to assume $B$ to be elliptic and Lipschitz continuous and we do not want to use $\sigma$. To this end we use Lemma 2.2 of Barles and Ramaswamy [6], which we recall within the proof of Proposition 2.1

Proof of Proposition 2.1. Let $\phi \in C^{2}(\overline{\mathcal{O}})$ and $\bar{x} \in \mathcal{O}$ be a local minimum of $w-\phi$, say in $\overline{B(\bar{x}, r)}$ for some $r>0$.

For all $\varepsilon>0$, we introduce the auxiliary function

$$
\Phi_{\varepsilon}(x, y)=u_{2}(x)-u_{1}(y)-\phi(x)+|x-y|^{2} / \varepsilon^{2}+|x-\bar{x}|^{4} .
$$

Let $\left(x_{\varepsilon}, y_{\varepsilon}\right)$ be the minimum point of $\Phi_{\varepsilon}(x, y)$ in $\overline{B(\bar{x}, r)} \times \overline{B(\bar{x}, r)}$. Since $\bar{x}$ is a strict local minimum point of $x \mapsto w(x)-\phi(x)+|x-\bar{x}|^{4}$, standard arguments show that

$$
\left(x_{\varepsilon}, y_{\varepsilon}\right) \rightarrow(\bar{x}, \bar{x}) \text { and }\left|x_{\varepsilon}-y_{\varepsilon}\right|^{2} / \varepsilon^{2} \rightarrow 0 \quad \text { as } \varepsilon \rightarrow 0 .
$$

Moreover, if $\zeta_{\varepsilon}(x, y):=\phi(x)-|x-y|^{2} / \varepsilon^{2}-|x-\bar{x}|^{4}$, we know that (cf. [13]), for every $\alpha>0$, there exist $X, Y \in \mathcal{S}^{n}(\mathbb{R})$ such that

$$
\begin{gathered}
\left(D_{x} \zeta_{\varepsilon}\left(x_{\varepsilon}, y_{\varepsilon}\right), X\right) \in \bar{J} \stackrel{2,-}{\mathcal{O}} u_{2}\left(x_{\varepsilon}\right), \quad\left(-D_{y} \zeta_{\varepsilon}\left(x_{\varepsilon}, y_{\varepsilon}\right), Y\right) \in \bar{J} \frac{2,+}{\mathcal{O}} u_{1}\left(y_{\varepsilon}\right), \\
-\left(1 / \alpha+\left\|D^{2} \zeta_{\varepsilon}\left(x_{\varepsilon}, y_{\varepsilon}\right)\right\|\right) \operatorname{Id} \leq\left(\begin{array}{cc}
-X & 0 \\
0 & Y
\end{array}\right) \leq-\left(\operatorname{Id}-\alpha D^{2} \zeta_{\varepsilon}\left(x_{\varepsilon}, y_{\varepsilon}\right)\right) D^{2} \zeta_{\varepsilon}\left(x_{\varepsilon}, y_{\varepsilon}\right),
\end{gathered}
$$

and

$$
\begin{array}{r}
F\left(X, D_{x} \zeta_{\varepsilon}\left(x_{\varepsilon}, y_{\varepsilon}\right), u_{2}\left(x_{\varepsilon}\right), x_{\varepsilon}\right) \leq 0, \\
F\left(Y,-D_{y} \zeta_{\varepsilon}\left(x_{\varepsilon}, y_{\varepsilon}\right), u_{1}\left(y_{\varepsilon}\right), y_{\varepsilon}\right) \geq 0 .
\end{array}
$$


The key point is to estimate

$$
F\left(X, D_{x} \zeta_{\varepsilon}\left(x_{\varepsilon}, y_{\varepsilon}\right), u_{2}\left(x_{\varepsilon}\right), x_{\varepsilon}\right)-F\left(Y,-D_{y} \zeta_{\varepsilon}\left(x_{\varepsilon}, y_{\varepsilon}\right), u_{1}\left(y_{\varepsilon}\right), y_{\varepsilon}\right)
$$

To this end we first choose $\alpha=\varepsilon^{2}$ and we use Lemma 2.2 in [6] which says the following : if the matrices $X, Y$ satisfy

$$
-\frac{\tilde{K}}{\varepsilon^{2}} \mathrm{Id} \leq\left(\begin{array}{cc}
-X & 0 \\
0 & Y
\end{array}\right) \leq \frac{\tilde{K}}{\varepsilon^{2}}\left(\begin{array}{cc}
\mathrm{Id} & -\mathrm{Id} \\
-\mathrm{Id} & \mathrm{Id}
\end{array}\right)
$$

then

$$
Y-X \leq-\frac{\tilde{K} \varepsilon^{2}}{6}(t X+(1-t) Y)^{2} \quad \text { for all } t \in[0,1]
$$

A slight modification of the arguments in [6] allows one to take into account the $D^{2} \phi$ and $D^{2}\left(|x-\bar{x}|^{4}\right)$ terms and yields

$$
Y-X^{\prime} \leq-\frac{\tilde{K} \varepsilon^{2}}{6}\left(t X^{\prime}+(1-t) Y\right)^{2}+O(\varepsilon) \quad \text { as } \varepsilon \rightarrow 0,
$$

for all $t \in[0,1]$, where $X^{\prime}=X+\left\|D^{2} \phi\right\| \operatorname{Id}+O\left(|x-\bar{x}|^{2}\right)$.

Now we are ready to estimate $F\left(X, p, u_{2}, x\right)-F\left(Y, q, u_{1}, y\right)$ with $p=D_{x} \zeta_{\varepsilon}\left(x_{\varepsilon}, y_{\varepsilon}\right)$ and $q=-D_{y} \zeta_{\varepsilon}\left(x_{\varepsilon}, y_{\varepsilon}\right)$. By using $(H 1)-(H 2)$ together with the inequality (2.11) for $t=0$, we get

$$
\begin{aligned}
0 \geq & F\left(X, p, u_{2}, x\right)-F\left(Y, q, u_{1}, y\right) \\
\geq & F\left(X^{\prime}, p, u_{1}, x\right)-F\left(Y, p, u_{1}, x\right) \\
& -K_{R}(|p-q|+|x-y|(|p|+|q|+\|Y\|))+\omega_{R}(|x-y|(1+|p|+|q|)) \\
& +\mathcal{M}_{\lambda, \Lambda}^{-}\left(\left\|D^{2} \phi\right\| \operatorname{Id}+O\left(|x-\bar{x}|^{2}\right)\right)+F\left(X, p, u_{2}, x\right)-F\left(X, p, u_{1}, x\right) \\
\geq & \kappa \frac{\tilde{K} \varepsilon^{2}}{6} \operatorname{tr}\left(Y^{2}\right)+O(\varepsilon)+\mathcal{M}_{\lambda, \Lambda}^{-}\left(\left\|D^{2} \phi\right\| \operatorname{Id}+O\left(|x-\bar{x}|^{2}\right)\right) \\
& -K_{R}(|p-q|+|x-y|(|p|+|q|+\|Y\|))+\omega_{R}(|x-y|(1+|p|+|q|)) \\
& +F\left(X, p, u_{2}, x\right)-F\left(X, p, u_{1}, x\right),
\end{aligned}
$$

where $R=\max \left(\left\|u_{1}\right\|_{\infty},\left\|u_{2}\right\|_{\infty}\right)$. In the last inequality, the "bad" term is $K_{R}|x-y|\|Y\|$ since the estimates on the test function $\phi$ do not ensure that this term converges to 0 . However, this term is controlled by the "good" term $\operatorname{tr}\left(Y^{2}\right)$ in the following way: by Cauchy-Schwarz's inequality,

$$
K|x-y|\|Y\| \geq-\kappa \frac{\tilde{K} \varepsilon^{2}}{6} \operatorname{tr}\left(Y^{2}\right)-O\left(|x-y|^{2} / \varepsilon^{2}\right) .
$$

And this estimate is now sufficient since we know that $|x-y|^{2} / \varepsilon^{2} \rightarrow 0$ as $\varepsilon \rightarrow 0$.

Thus by letting $\varepsilon \rightarrow 0$ we are led to

$$
\mathcal{M}^{-}\left(D^{2} \phi(\bar{x})\right)-b|D \phi(\bar{x})|+c(\bar{x}) w(\bar{x}) \leq 0,
$$


where $b=K_{R}$ and

$$
c(\bar{x})= \begin{cases}\frac{F\left(X, p, u_{2}(\bar{x}), \bar{x}\right)-F\left(X, p, u_{1}(\bar{x}), \bar{x}\right)}{u_{2}(\bar{x})-u_{1}(\bar{x})} & \text { if } u_{2}(\bar{x}) \neq u_{1}(\bar{x}), \\ 0 & \text { otherwise, }\end{cases}
$$

so the conclusion follows.

A fundamental tool in the theory of strong and viscosity solutions of elliptic equations is the Alexandrov-Bakelman-Pucci (ABP) estimate, a version of which we quote next.

Proposition 2.2. Let $\mathcal{O} \subset \mathbb{R}^{n}$ be a bounded domain and let $w \in C(\overline{\mathcal{O}})$ be a viscosity solution of

$$
\mathcal{M}_{\lambda, \Lambda}^{-}\left(D^{2} w\right)-b|D w|+c(x) w \leq f(x),
$$

where $b \in \mathbb{R}$ and $c, f \in L^{\infty}(\overline{\mathcal{O}})$. Suppose $c(x) \leq 0$ in $\mathcal{O}$. Then there exists a constant $C_{*}$, depending only on $\lambda, \Lambda,|b|$, and $\operatorname{diam}(\mathcal{O})$, such that

$$
\sup _{\mathcal{O}}(-w) \leq \sup _{\partial \mathcal{O}} w^{-}+C_{*}\|f\|_{L^{n}(\mathcal{O})} .
$$

Proof. This is a consequence of Proposition 2.12 in [11] (the proof of which is due to Trudinger). To link the notations in this paper with those in [11] we note that $\mathcal{M}_{\lambda, \Lambda}^{+}(X)=$ $-\mathcal{P}^{-}(X)$ and $\mathcal{M}_{\lambda, \Lambda}^{-}(X)=-\mathcal{P}^{+}(X)$, where

$$
\begin{aligned}
& \mathcal{P}^{-}(X)=-\Lambda \operatorname{tr}\left(X^{+}\right)+\lambda \operatorname{tr}\left(X^{-}\right), \\
& \mathcal{P}^{+}(X)=-\lambda \operatorname{tr}\left(X^{+}\right)+\lambda \operatorname{tr}\left(X^{-}\right),
\end{aligned}
$$

and $X^{+}, X^{-}$denote the positive and negative parts of $X \in \mathcal{S}^{n}(\mathbb{R})$.

It is trivial to deduce a maximum principle in small domains from the ABP inequality.

Proposition 2.3. Let $\mathcal{O} \subset \mathbb{R}^{n}$ be a bounded domain and suppose $b \geq 0$ and $c(x) \in$ $L^{\infty}(\overline{\mathcal{O}})$. There exists a constant $r>0$, depending on $\lambda, \Lambda, b$, $\operatorname{diam}(\mathcal{O}),\|c\|_{L^{\infty}(\overline{\mathcal{O}})}$, such that any viscosity solution $w \in C(\overline{\mathcal{O}})$ of

$$
\begin{cases}\mathcal{M}_{\lambda, \Lambda}^{-}\left(D^{2} w\right)-b|D w|+c(x) w \leq 0 & \text { in } \mathcal{O} \\ w \geq 0 & \text { on } \partial \mathcal{O}\end{cases}
$$

is nonnegative in $\mathcal{O}$, provided $|\mathcal{O}| \leq r$.

Proof. We apply Proposition 2.2 to

$$
\mathcal{M}_{\lambda, \Lambda}^{-}\left(D^{2} w\right)-b|D w|-c^{-}(x) w \leq-c^{+} w \leq c^{+} w^{-}
$$

and get

$$
\sup _{\mathcal{O}} w^{-} \leq C_{*}\left\|c^{+}\right\|_{L^{\infty}(\mathcal{O})}\left\|w^{-}\right\|_{L^{\infty}(\mathcal{O})}|\mathcal{O}|^{1 / n},
$$

from which the result follows. 
The following regularity result is a variation of the $C^{1, \alpha}$-regularity result proven by Caffarelli [9] in the case when $F$ depends only on $D^{2} u$ and by Święch [25] and Wang [26] in the general case of a proper equation, i.e. when $F$ is nonincreasing with respect to $u$.

Proposition 2.4. Let $\mathcal{O} \subset \mathbb{R}^{n}$ be a bounded domain and assume that $F$ satisfies (H1)$(H 2)$. Let $u \in C(\overline{\mathcal{O}})$ be a viscosity solution of

$$
F\left(D^{2} u, D u, u, x\right)=0 \quad \text { in } \mathcal{O} .
$$

Then $u \in C_{\text {loc }}^{1, \alpha}(\mathcal{O})$ for some $\alpha \in(0,1)$.

Proof. This theorem was proven in [25] under the supplementary assumptions that the constant $K_{R}$ in (H1)-(H2) is independent of $R$ and that $F$ is nonincreasing in $u$.

In order to reduce to the result of [25], suppose that the solution satisfies $|u| \leq K$. We set $F_{0}=F$ if $|u| \leq K, F_{0}=F(M, p, K, x)$ if $u>K$, and $F_{0}=F(M, p,-K, x)$ if $u<-K$. Then $F_{0}$ is globally Lipschitz with respect to $u$. We notice that $u$ is also a solution of $F_{0}\left(D^{2} u, D u, u, x\right)-k u=-k u \in C(\overline{\mathcal{O}})$. If $k$ is large enough the operator $F_{0}-k u$ is proper, thus we can apply the result in [25] to conclude the proof.

The next proposition asserts the existence of a principal eigenvalue and a principal eigenfunction for an operator without zero order term. We shall also use the fact that the principal eigenvalue goes to infinity as the measure of the domain goes to zero.

Proposition 2.5. Suppose $\mathcal{O}$ is a bounded smooth domain. Then there exists a number $\lambda_{1}=\lambda_{1}(\mathcal{O})>0$ and a function $\varphi_{1} \in C^{2}(\mathcal{O}) \cap C(\overline{\mathcal{O}})$ which satisfy

$$
\begin{cases}\mathcal{M}_{\lambda, \Lambda}^{-}\left(D^{2} \varphi_{1}\right)-b\left|D \varphi_{1}\right|+\lambda_{1} \varphi_{1}=0 & \text { in } \mathcal{O}, \\ \varphi_{1}>0 & \text { in } \mathcal{O}, \\ \varphi_{1}=0 & \text { on } \partial \mathcal{O} .\end{cases}
$$

In addition, we have $\lambda_{1}(\mathcal{O}) \rightarrow \infty$ as $|\mathcal{O}| \rightarrow 0$.

Proof. In the case $b=0$ this result was proved by Quaas in [18] (see also [8]). Essentially the same proof works for any $b$, since, by the known existence, uniqueness and regularity results for fully nonlinear operators (see [11], [25]) the operator $\mathcal{M}_{\lambda, \Lambda}^{-}\left(D^{2} \cdot\right)-b|D \cdot|$ has the same properties as $\mathcal{M}_{\lambda, \Lambda}^{-}\left(D^{2}\right.$.), namely, its inverse exists and is positivity preserving. Thus Krein-Rutman theory applies to the former just as to the latter.

We note that in a very recent work [20], [21], the authors established the existence of a principal eigenvalue and eigenfunction for any positively homogeneous operator, convex or concave in $D^{2} u$ and satisfying a condition of type (H3). In addition, in [21] a multitude of properties of these objects are proven, Proposition 2.5 being a very particular case of these.

We shall make use of a simple lemma, concerning products of viscosity solutions and test functions. 
Lemma 2.1. Let $u \in C(\mathcal{O}), u \geq 0$, satisfy

$$
\mathcal{M}_{\lambda, \Lambda}^{-}\left(D^{2} u\right)-b(x)|D u|+c(x) u \leq f(x) \quad \text { in } \mathcal{O},
$$

where $b, c, f \in L^{\infty}(\mathcal{O})$. Suppose $\psi \in C^{2}(\mathcal{O}) \cap C(\overline{\mathcal{O}})$ is strictly positive in $\overline{\mathcal{O}}$. Then $\bar{u}=u / \psi$ satisfies the inequality

$$
\mathcal{M}_{\lambda, \Lambda}^{-}\left(D^{2} \bar{u}\right)-\bar{b}(x)|D \bar{u}|+\bar{c}(x) \bar{u} \leq \bar{f}(x),
$$

where

$$
\bar{b}=b+2 \Lambda \sqrt{n} \psi^{-1}|D \psi|, \quad \bar{c}(x)=\frac{\mathcal{M}_{\lambda, \Lambda}^{-}\left(D^{2} \psi\right)-b|D \psi|}{\psi}+c(x), \quad \bar{f}=\frac{f}{\psi} .
$$

Proof. Suppose $u \in C^{2}(\mathcal{O})$, so that $\mathcal{M}_{\lambda, \Lambda}^{-}\left(D^{2} u\right)-b(x)|D u|+c(x) u \leq f(x)$ is satisfied in the classical sense. We have

$$
D u=\psi D \bar{u}+\bar{u} D \psi, \quad D^{2} u=\psi D^{2} \bar{u}+2 D \psi \otimes D \bar{u}+\bar{u} D^{2} \psi .
$$

Here and below, $\otimes$ denotes the symmetric tensor product, i.e. if $p, q \in \mathbb{R}^{n}$ then $p \otimes q=$ $\frac{1}{2}\left(p_{i} q_{j}+p_{j} q_{i}\right)_{i, j}$. By putting 2.16 into 2.14 and by using

$$
\mathcal{M}_{\lambda, \Lambda}^{-}(M+N) \geq \mathcal{M}_{\lambda, \Lambda}^{-}(M)+\mathcal{M}_{\lambda, \Lambda}^{-}(N), \quad \mathcal{M}_{\lambda, \Lambda}^{-}(\eta M)=\eta \mathcal{M}_{\lambda, \Lambda}^{-}(M),
$$

for $\eta \geq 0$, we obtain the statement of the lemma. Note that $\operatorname{tr}(A(p \otimes q)) \leq|A||p \otimes q| \leq$ $\sqrt{n} \Lambda|p||q|$, where $A$ is a matrix whose eigenvalues lie in $[\lambda, \Lambda]$, and $|A|:=\sqrt{\operatorname{tr}\left(A^{t} A\right)}$.

Let $u$ be only continuous. If (2.15) does not hold then there exist $x_{0} \in \mathcal{O}$ and $\phi \in$ $C^{2}(\mathcal{O})$ such that $\phi\left(x_{0}\right)=\bar{u}\left(x_{0}\right), \phi \leq \bar{u}$ in $\mathcal{O}$ and

$$
\mathcal{M}_{\lambda, \Lambda}^{-}\left(D^{2} \phi\left(x_{0}\right)\right)-\bar{b}\left(x_{0}\right)\left|D \phi\left(x_{0}\right)\right|+\bar{c}\left(x_{0}\right) \phi\left(x_{0}\right)>\bar{f}\left(x_{0}\right) .
$$

An easy computation then shows that this implies

$$
\mathcal{M}_{\lambda, \Lambda}^{-}\left(D^{2}(\phi \psi)\left(x_{0}\right)\right)-b\left|D(\phi \psi)\left(x_{0}\right)\right|+c\left(x_{0}\right) \phi\left(x_{0}\right) \psi\left(x_{0}\right)>f\left(x_{0}\right) .
$$

This contradicts 2.14), since $\phi \psi \in C^{2}(\mathcal{O})$ is a test function such that $\phi\left(x_{0}\right) \psi\left(x_{0}\right)=$ $u\left(x_{0}\right)$ and $\phi \psi \leq u$ in $\mathcal{O}$.

Next, we state a strong maximum principle for nonproper operators. In the literature there are more general results in the proper case, for example, a weak Harnack inequality is proven in [26], while in [5] a strong maximum principle is proven for degenerate operators.

Proposition 2.6. Let $\mathcal{O} \subset \mathbb{R}^{n}$ be a smooth domain and let $b, c \in L^{\infty}(\overline{\mathcal{O}})$. Suppose $w \in C(\overline{\mathcal{O}})$ is a viscosity solution of

$$
\begin{cases}\mathcal{M}_{\lambda, \Lambda}^{-}\left(D^{2} w\right)-b(x)|D w|+c(x) w \leq 0 & \text { in } \mathcal{O} \\ w \geq 0 & \text { in } \mathcal{O} .\end{cases}
$$


Then either $w \equiv 0$ in $\mathcal{O}$ or $w>0$ in $\mathcal{O}$ and at any point $x_{0} \in \partial \mathcal{O}$ at which $w\left(x_{0}\right)=0$ we have

$$
\liminf _{t \searrow 0} \frac{w\left(x_{0}+t v\right)-w\left(x_{0}\right)}{t}>0,
$$

where $v \in \mathbb{R}^{n} \backslash 0$ is such that $v \cdot n(x)<0$; here $n(x)$ denotes the exterior normal to $\partial \mathcal{O}$ at $x_{0}$.

Proof. If $c(x) \leq 0$ in $\mathcal{O}$ this follows from Theorems 1 and 2 in [5]. Note that in that paper the operator $F$ is supposed to be continuous in $x$, but the arguments there are very easy to adapt to an operator like the one in 2.18 , in which the first order and zero order coefficients are only measurable and bounded.

By using Proposition 2.5 and Lemma 2.1 we can show that we can always reduce the problem to a proper one, that is, to a problem in which $c(x) \leq 0$. Indeed, suppose $x_{0} \in \mathcal{O}$ is a point at which $w\left(x_{0}\right)=0$. By Proposition 2.5 there exists a sufficiently small ball $B$ around $x_{0}$ such that the first eigenvalue of $\mathcal{M}_{\lambda, \Lambda}^{-}\left(D^{2} \cdot\right)-b|D \cdot|$ in this ball is larger than the $L^{\infty}$-norm of $c$. By setting $\psi=\varphi_{1}\left(\varphi_{1}\right.$ is the first eigenfunction of this operator in $B$, see Proposition 2.5 and by applying Lemma 2.1 in a smaller concentric ball $B_{1} \subset B$ we obtain a proper equation for $u / \psi$ in $B_{1}$. Then from the result in [5] it follows that $u \equiv 0$ in the small ball. This means each point in $\mathcal{O}$ at which $u$ vanishes has a neighbourhood in which $u$ is identically zero, so $u$ vanishes everywhere in $\mathcal{O}$. We argue in a similar way if $x_{0} \in \partial \mathcal{O}$ and we conclude the proof.

We are now ready to show that the arguments of [7] adapt to our setting, and permit us to prove Theorem 1.1 and, in a similar manner, that the arguments from [24] can be used to prove Theorems 1.2 and 1.3 .

Proof of Theorem 1.1. The following argument, due to Berestycki and Nirenberg, is given here for completeness. Suppose $\mathcal{O}$ is convex in the direction of the vector $e_{1}=$ $(1,0, \ldots, 0)$ and is symmetric with respect to the hyperplane $T_{0}=\left\{x \mid x_{1}=0\right\}$. We want to show that

$$
u\left(-x_{1}, x_{2}, \ldots, x_{N}\right)=u\left(x_{1}, x_{2}, \ldots, x_{N}\right) \quad \text { for any } x \in \mathcal{O} .
$$

For any $\lambda \in \mathbb{R}$ we define

$$
\begin{aligned}
T_{\lambda} & =\left\{x \mid x_{1}=\lambda\right\}, \quad D_{\lambda}=\left\{x \mid x_{1}>\lambda\right\}, \quad \Sigma_{\lambda}=D_{\lambda} \cap \mathcal{O}, \\
x^{\lambda} & =\left(2 \lambda-x_{1}, x_{2}, \ldots, x_{n}\right), \quad \text { the reflexion of } x \text { with respect to } T_{\lambda}, \\
v_{\lambda}(x) & =u\left(x^{\lambda}\right), \quad w_{\lambda}(x)=u\left(x^{\lambda}\right)-u(x), \quad \text { for } x \in \Sigma_{\lambda}, \\
d & =\inf \left\{\lambda \in \mathbb{R} \mid T_{\mu} \cap \overline{\mathcal{O}}=\emptyset \text { for all } \mu>\lambda\right\} .
\end{aligned}
$$

With this notation, our goal is to show that $w_{0} \equiv 0$ in $\Sigma_{0}$.

By hypothesis $\left(O_{1}\right)$ the function $v_{\lambda}$ satisfies the same equation as $u$. Hence, by Proposition 2.1. $w_{\lambda}$ satisfies

$$
L_{\lambda} w_{\lambda}:=\mathcal{M}_{\lambda, \Lambda}^{-}\left(D^{2} w_{\lambda}\right)-b\left|D w_{\lambda}\right|+c_{\lambda}(x) w_{\lambda} \leq 0 \quad \text { in } \Sigma_{\lambda},
$$

where $c_{\lambda}$ is a measurable function which is bounded independently of $\lambda$. 
We say the hyperplane $T_{\lambda}$ has reached a position $\lambda<d$ provided $w_{\mu}$ is nonnegative in $\Sigma_{\mu}$ for all $\mu \in[\lambda, d)$. The plane $T_{\lambda}$ "starts" at $\lambda=d$ and "moves" to the left as $\lambda$ decreases. If we prove that $T_{\lambda}$ reaches position zero we are done, since then we can take a hyperplane coming from the other side, that is, starting from $-d$ and moving to the right. The situation is totally symmetric so the second hyperplane would also reach position zero. This means that $w_{0} \geq 0$ and $w_{0} \leq 0$ in $\Sigma_{0}$, hence $w_{0} \equiv 0$ in $\Sigma_{0}$.

We first show that the above procedure can begin, that is, there exists $\bar{\lambda}<d$ such that $w_{\mu} \geq 0$ in $\Sigma_{\mu}$ for all $\mu \in[\bar{\lambda}, d)$. By using Proposition 2.3 we can find a number $r>0$ such that the operator $L_{\lambda}$ defined above satisfies the maximum principle in any subdomain $\mathcal{O}^{\prime} \subset \mathcal{O}$ with $\left|\mathcal{O}^{\prime}\right|<r$. We fix $\bar{\lambda}<d$ so close to $d$ that $\left|\Sigma_{\lambda}\right|<r$ for any $\lambda \in[\bar{\lambda}, d)$. Hence, by Proposition 2.3 inequality 2.19 implies that $w_{\mu} \geq 0$ in $\Sigma_{\mu}$ for all $\mu \in[\bar{\lambda}, d)$.

Note that, by the definition of $w_{\lambda}$, we have $w_{\lambda}>0$ on $\partial \Sigma_{\lambda} \cap \partial \mathcal{O}$ for any $\lambda \in$ $(0, d)$ (since $u$ vanishes on $\partial \mathcal{O}$ and is strictly positive in $\mathcal{O}$ ). Hence, by Hopf's lemma (Proposition 2.6), $w_{\lambda}>0$ in $\Sigma_{\lambda}$ for $\lambda \in(\bar{\lambda}, d)$.

We can define the number

$$
\lambda_{0}=\inf \left\{\lambda \geq 0 \mid w_{\mu} \geq 0 \text { in } \Sigma_{\mu} \text { for all } \mu \geq \lambda\right\} .
$$

Note that, by continuity with respect to $\lambda, w_{\lambda_{0}} \geq 0$ in $\Sigma_{\lambda_{0}}$. By Hopf's lemma, if $\lambda_{0}>0$ then $w_{\lambda_{0}}>0$ in $\Sigma_{\lambda_{0}}$. Further, we claim that $\partial u / \partial x_{1}<0$ in $\Sigma_{\lambda_{0}}$ (recall that $u \in C^{1}$, by Proposition 2.4). Indeed, let $x$ be an arbitrary point in $\Sigma_{\lambda_{0}}$ with $x_{1}=\lambda>\lambda_{0}$. Then, by the preceding remarks, $w_{\lambda}>0$ in $\Sigma_{\lambda}$. Since $w_{\lambda}=0$ on $T_{\lambda}$, Proposition 2.6 implies

$$
0<\frac{\partial w_{\lambda}}{\partial x_{1}}(x)=-2 \frac{\partial u}{\partial x_{1}}(x)
$$

(recall that $\left.w_{\lambda}(x)=u\left(x^{\lambda}\right)-u(x)\right)$.

Suppose for contradiction $\lambda_{0}>0$. We are going to "push" the moving plane to the left of $\lambda_{0}$. Let $K$ be a compact subset of $\Sigma_{\lambda_{0}}$ such that $\left|\Sigma_{\lambda_{0}} \backslash K\right|<r / 2$ ( $r$ is the number from Proposition 2.3. Since $w_{\lambda_{0}}$ is continuous and strictly positive in $\Sigma_{\lambda_{0}}$, there exists a number $\varepsilon>0$ such that $w_{\lambda_{0}} \geq \varepsilon$ in $K$. Fix a number $\lambda_{1}$ with $0<\lambda_{1}<\lambda_{0}$ such that $\left|\Sigma_{\lambda} \backslash K\right|<\delta$ for $\lambda \in\left[\lambda_{1}, \lambda_{0}\right)$. By continuity, if $\lambda_{1}$ is sufficiently close to $\lambda_{0}$ we have $w_{\lambda} \geq \varepsilon / 2>0$ in $K$ for any $\lambda \in\left[\lambda_{1}, \lambda_{0}\right)$. In the remaining part of $\Sigma_{\lambda}$ the function $w_{\lambda}$, $\lambda \in\left[\lambda_{1}, \lambda_{0}\right)$, satisfies the equation

$$
\begin{cases}L_{0} w_{\lambda} \leq 0 & \text { in } \Sigma_{\lambda} \backslash K \\ w_{\lambda_{1}} \geq 0 & \text { on } \partial\left(\Sigma_{\lambda} \backslash K\right) .\end{cases}
$$

By Proposition 2.3. $w_{\lambda} \geq 0$ in $\Sigma_{\lambda} \backslash K$. Hence $w_{\lambda} \geq 0$ in $\Sigma_{\lambda}$ for any $\lambda \in\left[\lambda_{1}, \lambda_{0}\right)$. This contradicts the definition of $\lambda_{0}$.

Proof of Theorem 1.3. Now we define $\Sigma_{\lambda}=\left\{x_{1}>\lambda\right\} \backslash B^{\lambda}$, where $B^{\lambda}$ is the reflexion of $B$ with respect to $T_{\lambda}$. As before the difference function $w_{\lambda}$ is defined in $\Sigma_{\lambda}$ and we need to show that $w_{0} \geq 0$.

Let us prove that for sufficiently large $\lambda$ we have $w_{\lambda} \geq 0$ in $\Sigma_{\lambda}$. Suppose this is not true, that is, there exists a sequence $\lambda_{m} \rightarrow \infty$ such that $w_{\lambda_{m}}$ takes negative values in $\Sigma_{\lambda_{m}}$. 
Let $m$ be large enough so that $0<u<a=\left.u\right|_{\partial B}$ in $D_{\lambda_{m}}$. Take $x^{(m)} \in \Sigma_{\lambda_{m}}$ to be such that

$$
w_{\lambda_{m}}\left(x^{(m)}\right)=\min _{\Sigma_{\lambda_{m}}} w_{\lambda_{m}}<0
$$

(the minimum is clearly attained for fixed $m$ since $w_{\lambda_{m}} \geq 0$ on $\partial \Sigma_{\lambda_{m}}$ and $w_{\lambda_{m}} \rightarrow 0$ as $\left.|x| \rightarrow \infty, x \in \Sigma_{\lambda_{m}}\right)$. Note that $0<u\left(x^{\lambda}\right)<u(x)$ whenever $w_{\lambda}(x)<0$ so that for $m$ large enough each point $x^{(m)}$ has a neighbourhood $\mathcal{U}_{m}$ in which both $u(x), u\left(x^{\lambda}\right) \in$ $(0, \delta)$ (since $u \rightarrow 0$ at infinity), where $\delta$ is the number from Theorem 1.3 . Hence in this neighbourhood $c_{\lambda_{m}} \leq 0$, by Proposition 2.1, and

$$
\mathcal{M}_{\lambda, \Lambda}^{-}\left(D^{2} w_{\lambda_{m}}\right)-b\left|D w_{\lambda_{m}}\right| \leq 0 \quad \text { in } \mathcal{U}_{m}
$$

Since $w_{\lambda_{m}}$ attains an interior minimum in $\mathcal{U}_{m}$, we obtain a contradiction with Proposition 2.6. applied to the last inequality.

This reasoning shows that we can define the critical position $\lambda_{0}$ as before. We again aim to show that $\lambda_{0}=0$. Note that, as in the bounded domain case, we have

$$
\frac{\partial u}{\partial x_{1}}<0 \quad \text { in } \Sigma_{\lambda_{0}}
$$

First, we claim that $\lambda_{0} \leq R$, where $R$ is the radius of $B$. Suppose this is not true. Then there exist sequences $\lambda_{m}$ and $x^{(m)} \in \Sigma_{\lambda_{m}}$ such that $R<\lambda_{m} \leq \lambda_{0}, \lambda_{m} \rightarrow \lambda_{0}$, and $w_{\lambda_{m}}$ attains its negative minimum in $\bar{\Sigma}_{\lambda_{m}}$ at $x^{(m)}$.

The point is that $x^{(m)}$ cannot be on the boundary of $\Sigma_{\lambda_{m}}$. Indeed, note that $\partial \Sigma_{\lambda_{m}}$ is regular, $\partial \Sigma_{\lambda_{m}}=T_{\lambda_{m}} \cup \partial B^{\lambda_{m}}$, and write $\partial B^{\lambda_{m}}=S_{l} \cup S_{r}$, where $S_{l}$ (the left part of $\partial B^{\lambda_{m}}$ ) contains the points on $\partial B^{\lambda_{m}}$ which are such that starting from these points and moving to the left along the direction $-x_{1}$ one enters $\Sigma_{\lambda_{m}}$. Each point $z \in S_{r}$ has its counterpart $\bar{z} \in S_{l}$ with $z^{\prime}=\bar{z}^{\prime}$, where for all $z \in \mathbb{R}^{n}, z^{\prime}=\left(z_{2}, \ldots, z_{n}\right)$. Note that, by 2.20,

$$
w_{\lambda_{m}}(z)=a-u(z)>a-u(\bar{z})=w_{\lambda_{m}}(\bar{z}), \quad z \in S_{r},
$$

so $w_{\lambda_{m}}$ does not attain its minimum on $S_{r}$. On the other hand, by 2.20 and hypothesis (1.7), for any $z \in S_{l}$ the difference $w_{\lambda_{m}}$ decreases strictly to the left of $z$, so that $w_{\lambda_{m}}$ does not attain its minimum on $S_{l}$ either.

Further, as above we can show that $x^{(m)}$ cannot tend to infinity, so $x^{(m)} \rightarrow x_{0} \in \bar{\Sigma}_{\lambda_{0}}$, where $x_{0}$ is such that $w_{\lambda_{0}}\left(x_{0}\right)=0$ and $D w_{\lambda_{0}}\left(x_{0}\right)=0$. This contradicts Proposition 2.6 . applied to $w_{\lambda_{0}} \geq 0$.

We have shown that the moving plane "enters" $B$. In particular, repeating this argument for all directions, it follows from 2.20 that each point $x \in \partial B$ has a neighbourhood $\mathcal{U}_{x}$ such that $u$ is strictly decreasing in $\mathcal{U}_{x}$ along all directions which make an acute angle with $x$. This implies that if $T_{\lambda}$ meets $\partial B$ at $x$, then $w_{\lambda}$ is positive in $\mathcal{U}_{x} \cap \Sigma_{\lambda}$ for any $\lambda>0$.

Suppose now $0<\lambda_{0} \leq R$. We again take sequences $\lambda_{m} \rightarrow \lambda_{0}$ and $x^{(m)}$ at which $w_{\lambda_{m}}$ attains its negative minimum. In order to show that $x^{(m)}$ cannot be on the boundary of $\Sigma_{\lambda_{m}}$ we now have to distinguish three types of points on $\partial B^{\lambda_{m}}$ : the two types considered above, which are treated in the same way, and the points $z \in \partial B^{\lambda_{m}}$ which are such that if one starts from $z$ and moves to the left along $-x_{1}$ one enters $B^{\lambda_{m}}$ and meets $\partial B$ before 
meeting $T_{\lambda_{m}}$. Since $u=a$ on $\partial B$, for any such $z$ we have $u(z)<a$, by 2.20 , so $w_{\lambda_{m}}(z)>0$.

Hence $x^{(m)} \rightarrow x_{0} \in \bar{\Sigma}_{\lambda_{0}}$. If $x_{0}$ belongs to the regular part of $\Sigma_{\lambda_{0}}$ we have a contradiction with Proposition 2.6. If $x_{0}$ is on the singular part of $\Sigma_{\lambda_{0}}$, that is, $x_{0}$ is a point at which $T_{\lambda_{0}}$ meets $\partial B$, we have a contradiction with the positivity of the comparison functions in a neighbourhood of $\partial B$, for sufficiently large $m$.

Proof of Theorem 1.2 We use exactly the same argument as for Theorem 1.3 (this argument is now considerably simpler) replacing $\partial B$ by a point of local maximum of $u$. It then follows that $u$ is radially symmetric with respect to this point.

\section{References}

[1] Alexandrov, A. D.: A characteristic property of spheres. Ann. Mat. Pura Appl. 58, 303-315 (1962) Zbl 0107.15603 MR 0143162

[2] Badiale, M.: Geometrical properties of fully nonlinear equations and an application to singularities. J. Differential Equations 112, 33-52 (1994) Zbl 0804.35013 MR 1287551

[3] Badiale, M., Bardi, M.: Symmetry properties of solutions of Hamilton-Jacobi equations without uniqueness. Nonlinear Anal. 15, 1031-1043 (1990) Zbl 0736.35020 MR 1082279

[4] Badiale, M., Bardi, M.: Asymptotic symmetry of solutions of nonlinear partial differential equations. Comm. Pure Appl. Math. 45, 899-921 (1992) Zbl 0801.35015 MR 1164068

[5] Bardi, M., Da Lio F. : On the strong maximum principle for fully nonlinear degenerate elliptic equations. Arch. Math. (Basel) 73, 276-285 (1999) Zbl 0939.35038 MR 1710100

[6] Barles, G., Ramaswamy, M.: Sufficient structure conditions for uniqueness of viscosity solutions of semilinear and quasilinear equations. NoDEA Nonlinear Differential Equations Appl. 12, 203-523 (2005) Zbl pre05068179 MR 2199386

[7] Berestycki, H., Nirenberg, L.: On the method of moving planes and the sliding method. Bull. Soc. Brazil Mat. Nova Ser. 22, 1-37 (1991) Zbl 0784.35025 MR 1159383

[8] Busca, J., Esteban, M., Quaas, A.: Nonlinear eigenvalues and bifurcation problems for Pucci's operators. Ann. Inst. H. Poincaré Anal. Non Linéaire 22, 187-206 (2005) Zbl pre02165098 MR 2124162

[9] Caffarelli, L.: Interior a priori estimates for solutions of fully nonlinear equations. Ann. of Math. 130, 189-213 (1989) Zbl 0692.35017 MR 1005611

[10] Caffarelli, L., Cabre, X.: Fully Nonlinear Elliptic Equations. Colloq. Publ. 43, Amer. Math. Soc. (1995) Zbl 0834.35002 MR 1351007

[11] Caffarelli, L. Crandall, M. G., Kocan, M., Święch, A.: On viscosity solutions of fully nonlinear equations with measurable ingredients, Comm. Pure Appl. Math. 49, 365-397 (1996) Zbl 0854.35032 MR 1376656

[12] Crandall, M. G., Kocan, M., Lions, P. L., Święch, A.: Existence results for boundary problems for uniformly elliptic and parabolic fully nonlinear equations. Electron. J. Differential Equations 1999, no. 24, 22 pp. Zbl 0927.35029 MR 1696765

[13] Crandall, M. G., Ishii, H., Lions, P. L.: User's guide to viscosity solutions of second order partial differential equations. Bull. Amer. Math. Soc. 27, 1-67 (1992) Zbl 0755.35015 MR 1118699

[14] Felmer, P., Quaas, A.: Positive solutions to a "semilinear" equation involving the Pucci's operator. J. Differential Equations 199, 376-393 (2004) Zbl 1070.34032 MR 2047915 
[15] Gidas, B., Ni, W.-M., Nirenberg, L.: Symmetry and related properties via the maximum principle. Comm. Math. Phys. 68, 209-243 (1979) Zbl 0425.35020 MR 0544879

[16] Li, C.: Monotonicity and symmetry of solutions of fully nonlinear elliptic equations. Comm. Partial Differential Equations 16, 491-526 and 585-615 (1991) Zbl 0741.35014 MR 1113099

[17] Pucci, C.: Operatori ellittici estremanti. Ann. Mat. Pura Appl. 72, 141-170 (1966) Zbl 0154.12402 MR 0208150

[18] Quaas, A.: Existence of positive solutions to a "semilinear" equation involving Pucci's operator in a convex domain, Differential Integral Equations 17, 481-494 (2004) MR 2054930

[19] Quaas, A., Sirakov, B.: Existence results for nonproper elliptic equations involving the Pucci operator. Comm. Partial Differential Equations 31, 987-1003 (2006) Zbl pre05062563 MR 2254600

[20] Quaas, A., Sirakov, B.: On the principal eigenvalue and the Dirichlet problem for fully nonlinear operators. C. R. Math. Acad. Sci. Paris 342, 115-118 (2006) Zbl pre02248352 MR 2193657

[21] Quaas, A., Sirakov, B.: Existence and properties of a principal eigenvalue of fully nonlinear elliptic operators. Preprint

[22] Reichel, W.: Radial symmetry for elliptic boundary-value problems on exterior domains. Arch. Ration. Mech. Anal. 137, 381-394 (1997) Zbl 0891.35006 MR 1463801

[23] Serrin, J.: A symmetry theorem in potential theory. Arch. Ration. Mech. Anal. 43, 304-318 (1971) Zbl 0222.31007 MR 0333220

[24] Sirakov, B.: Symmetry for exterior elliptic problems and two conjectures in potential theory. Ann. Inst. H. Poincaré Anal. Non Linéaire 18, 135-156 (2001) Zbl 0997.35014 MR 1808026

[25] Święch, A.: $W^{1, p}$-interior estimates for solutions of fully nonlinear, uniformly elliptic equations. Adv. Differential Equations 2, 1005-1027 (1997) Zbl 1023.35509 MR 1606359

[26] Wang, L.:, On the regularity theory of fully nonlinear parabolic equations: I, II. Comm. Pure Appl. Math. 45, 27-76 (1992) Zbl 0832.35025 MR 1135923 